\title{
Introduction
}

\section{LES FEMMES CONTESTENT}

Genre, féminismes et mobilisations collectives

Laure Bereni, Anne Revillard

Presses de Sciences Po (P.F.N.S.P.)| «Sociétés contemporaines »

2012/1 n $85 \mid$ pages 5 à 15

ISSN 1150-1944

ISBN 9782724632750

\section{Article disponible en ligne à l'adresse :}

https://www.cairn.info/revue-societes-contemporaines-2012-1-page-5.htm

\section{Pour citer cet article :}

Laure Bereni, Anne Revillard « Les femmes contestent. Genre, féminismes et mobilisations collectives », Sociétés contemporaines 2012/1 (n85), p. 5-15. DOI 10.3917/soco.085.0005

Distribution électronique Cairn.info pour Presses de Sciences Po (P.F.N.S.P.).

(C) Presses de Sciences Po (P.F.N.S.P.). Tous droits réservés pour tous pays.

La reproduction ou représentation de cet article, notamment par photocopie, n'est autorisée que dans les limites des conditions générales d'utilisation du site ou, le cas échéant, des conditions générales de la licence souscrite par votre établissement. Toute autre reproduction ou représentation, en tout ou partie, sous quelque forme et de quelque manière que ce soit, est interdite sauf accord préalable et écrit de l'éditeur, en dehors des cas prévus par la législation en vigueur en France. Il est précisé que son stockage dans une base de données est également interdit. 

Introduction

e dossier, issu de plusieurs années de réflexions collectives
au sein du séminaire "Genre, féminismes et mobilisations
collectives » à Paris ${ }^{1}$, présente quelques apports de l'étude
d'une catégorie particulière de mouvements sociaux, les mouvements
des femmes et féministes, à la sociologie des protestations collectives.

Les recherches portant sur ces mouvements, que l'on peut définir comme les mobilisations collectives menées principalement par des femmes, en tant que femmes (mouvements des femmes) et/ou contestant la hiérarchie socialement établie entre les sexes (mouvements féministes) ${ }^{2}$, n'offrent pas seulement un apport empirique au champ de la sociologie des mouvements sociaux. L'exploration de ces mobilisations depuis plusieurs décennies, en premier lieu en langue anglaise et dans le monde académique américain, a proposé des clés analytiques pour critiquer et enrichir les concepts de la sociologie des mouvements sociaux. Ce dossier s'attache à présenter, dans le contexte français, l'impact de certains acquis de la recherche anglophone sur les mouvements des femmes et féministes (cf. la revue de la littérature de L. Bereni et A. Revillard, et la traduction d'un texte de la sociologue américaine M. Katzenstein) et rend compte des travaux récents qui, menés sur des terrains français, croisent les apports de ce riche champ d'analyse anglophone, les perspectives de la sociologie du militantisme développée en France, et celles des études sur le genre (cf. les articles de M. Charpenel, A. Jacquemart et B. Pavard). Cette démarche nous paraît d'autant plus importante que les mouvements des femmes et féministes restent empiriquement très mal connus en France - ayant été peu

1/ Séminaire qui s'est tenu mensuellement depuis 2004 à l'École des Hautes Études en Sciences Sociales à Paris, soutenu par l'École Normale Supérieure et l'association EFiGiES (Association de jeunes chercheuses et chercheurs en études féministes, genre et sexualités).

2/ Pour une définition plus détaillée de cette catégorie, arguant de l'intérêt analytique de réunir dans une même classe d'objets les mouvements féminins et féministes, cf. (Bereni, Revillard, dans ce numéro). Voir aussi (Ferree, Mueller, 2004 ; Mazur, McBride, 2008). 
étudiés jusqu'à une période récente - et que les contributions théoriques de la littérature anglophone sur cet objet y sont encore insuffisamment diffusées.

Récemment, plusieurs articles, ouvrages et numéros spéciaux de revues francophones ont tiré le bilan de deux décennies de riches développements en sociologie du militantisme et des protestations collectives, soulignant les perspectives originales, tant méthodologiques que théoriques, développées en France depuis une vingtaine d'années par rapport aux paradigmes dominants du champ académique américain (Sawicki, Siméant, 2009 ; Agrikoliansky, Fillieule, Sommier, 2010 ; Combes, Hmed, Mathieu, Siméant, Sommier, 2011). Il est reproché au champ anglo-saxon, dont les auteurs de Dynamics of Contention (McAdam, Tarrow, Tilly, 2001) constituent des figures tutélaires, d'être de plus en plus clos sur lui-même, et de céder à une routinisation méthodologique et conceptuelle qui le conduit à un assèchement analytique - même si ses importants apports théoriques sont appréciés et mobilisés en France. Ces critiques soulignent que les recherches déployées dans le champ français - qui, faut-il le rappeler, sont largement dominées à l'échelle internationale - s'appuient sur des protocoles méthodologiques plus souples et ethnographiques, sont plus attentives à l'échelle microsociologique et au temps court, opèrent davantage de croisements avec l'histoire, l'anthropologie et la science politique, et articulent plus étroitement l'analyse des mouvements sociaux avec celle du militantisme «traditionnel » (partisan, syndical...). Mais comme le mentionnent par ailleurs ces bilans, le champ français n'est pas non plus exempt de rigidités, de biais et de vides analytiques. La sociologie française des mobilisations collectives et du militantisme s'est, par exemple, focalisée d'abord sur les logiques individuelles de l'engagement au détriment d'analyses plus méso ou macrosociologiques, et a porté l'essentiel de son attention sur des mouvements de gauche au détriment, notamment, des mobilisations conservatrices ou se présentant comme apolitiques.

La comparaison réflexive des champs d'étude des mobilisations collectives en France et aux États-Unis, et en particulier des statuts différentiels qu'y occupent les travaux sur les mouvements des femmes et féministes, a constitué l'un des points de départ de ce dossier.

Aux États-Unis, les mouvements des femmes et féministes constituent des objets canoniques de la sociologie des mouvements sociaux. Dès les années 1970, alors que ce domaine d'étude prenait 
son essor aux États-Unis en renouvelant ses paradigmes et ses méthodes, le mouvement féministe, qui avait particulièrement marqué les contestations sociales et politiques des années 1960 (aux côtés des mobilisations pour les droits civiques, environnementales, étudiantes...), s'est imposé assez vite comme l'un des terrains privilégiés de ce nouveau champ de recherche. Les travaux sur le mouvement de libération des femmes américain menés par Jo Freeman, l'une des rares sociologues femmes à figurer alors parmi les théoriciens reconnus des mouvements sociaux, ont été d'emblée intégrés dans les discussions conceptuelles de ce champ d'étude. Tout en s'inscrivant dans le paradigme dominant de la «mobilisation des ressources $^{3} »$ (McCarthy, Zald, 1977), Jo Freeman défendait l'importance d'une approche généalogique des mouvements sociaux. Elle montrait notamment que les transformations affectant les institutions politiques dominantes (féminisation des élites bureaucratiques et politiques, mise en place d'un cadre juridique sanctionnant les discriminations selon le sexe...) avaient été déterminantes dans l'essor du mouvement féministe de la seconde vague ${ }^{4}$ (Freeman, 1973 ; 1975). Dans les décennies suivantes, alors que le monde académique américain se féminisait et que les études féministes et sur le genre acquéraient une légitimité institutionnelle croissante, l'étude des mouvements des femmes a alimenté et déstabilisé les principaux paradigmes d'analyse des mouvements sociaux. Ces recherches ont notamment participé à la construction du paradigme du « processus politique $\gg^{5}$, qui s'est imposé dans les années 1980 à la suite des travaux de Tilly (Tilly, 1978) et de McAdam (McAdam, 1982), mais aussi à sa critique, en pointant le biais statique de ce modèle, et en invitant à placer le regard sur la manière dont les mouvements et institutions se façonnent mutuellement à travers le temps (Costain, 1992 ; Banaszak, Beckwith, Rucht, 2003).

L'étude des mouvements féministes a ainsi été à l'origine de plusieurs innovations conceptuelles importantes. C'est, par exemple, à partir d'une exploration socio-historique des mouvements des femmes aux États-Unis que Verta Taylor a forgé le concept de

3/ Ce courant théorique, qui a pris son essor dans les années 1970 aux États-Unis, envisage l'action collective comme résultant d'entreprises rationnelles de mobilisations de diverses ressources par des « organisations de mouvement social ».

4/ L'historiographie des mouvements féministes dans le monde occidental distingue classiquement une «première vague » de mobilisation entre la fin du XIX et le milieu du XX siècle, principalement autour de la revendication du droit de suffrage, puis une «deuxième vague » qui émerge dans les années 1960 et 1970, avec des mouvements centrés sur la politisation du « privé » (libre accès à la contraception et l'avortement, dénonciation des violences et inégalités logés dans les rapports familiaux ou intimes...) et dominés par des rhétoriques radicales.

5/ Le paradigme du processus politique accorde une importance centrale à l'environnement politique pour comprendre le développement des mobilisations contestataires. 
«structures dormantes» (abeyance structures), pour désigner les organisations qui, dans les périodes de « creux » des cycles de mobilisation, constituent des lieux de maintien de discours, de ressources organisationnelles et de répertoires d'action qui peuvent être réactivés lors de mobilisations futures, et tracent des continuités entre deux cycles (ou «vagues») de mobilisation (Taylor, 2005). C'est également à partir de l'étude des mouvements féministes qu'a été forgée la catégorie de «communauté de mouvement social», qui désigne la configuration des individus et des organisations (politiques, culturelles, sociales...) réunis par leur allégeance à des visions du monde communes (ici, féministes), à l'opposé d'une définition des mouvements sociaux comme composés d'organisations structurées et principalement orientées vers des buts politiques (Taylor \& Whittier, 1992 ; Staggenborg, 1998). Enfin, c'est en analysant les mobilisations féministes à l'intérieur de l'armée et de l'Église catholique américaine depuis les années 1970 que Mary Katzenstein a montré que la contestation peut se déployer de manière invisible (unobtrusive), à l'intérieur des institutions, au sein de ce qu'elle appelle des «habitats institutionnels»- une perspective qui la conduit à contester la frontière rigide habituellement tracée par les analystes des mouvements sociaux entre insiders et outsiders (Katzenstein, 1998) ${ }^{6}$. Ainsi, dans le champ très prolifique de la sociologie des mouvements sociaux aux États-Unis, les mouvements des femmes ont été largement travaillés, conduisant à des critiques et renouvellements conceptuels qui ont globalement été intégrés dans les questionnements et débats de ce champ.

Par contraste, en France, les mouvements des femmes et féministes sont restés, jusqu'aux dernières années de la décennie 2000, des objets sous-investis dans le champ d'étude sur les mouvements sociaux, pourtant très dynamique depuis une vingtaine d'années. Les «bonnes causes» de la sociologie américaine des mouvements sociaux ne sont pas celles de la sociologie française. En France, les objets canoniques de ce domaine d'étude ont été, en premier lieu, les mobilisations de groupes à faibles ressources, couramment unifiés dans la catégorie des «sans»- sans papiers, sans emploi, sans logis (Siméant, 1998; Maurer, 2001 ; Dunezat, 2004; Péchu, 2004 ; Mouchard, 2009) -, puis le mouvement altermondialiste (Agrikoliansky, Fillieule, Mayer, 2005 ; Agrikoliansky, Sommier, 2005 ; Mouchard, 2005). L'étude des mouvements des femmes a longtemps été placée dans un régime d'exceptionnalité, relevant du prophétisme théorique (Touraine, 1982), de l'histoire faite par

6/ Des extraits des deux premiers chapitres de Faithful and Fearless, de Mary Katzenstein (1998), sont traduits dans ce numéro. Dans la même perspective voir aussi (Banaszak, 2010). 
d'anciennes actrices du mouvement (Garcia Guadilla, 1981 ; Picq, 1993) ou de la discipline historique (Klejman, Rochefort, 1989 ; Bard, 1995 ; Gubin, et al., 2004), sans parvenir à s'imposer comme un terrain de constitution et d'épreuve sociologique des paradigmes du champ ${ }^{7}$. Ce n'est pas l'absence de matériaux empiriques qui est en cause : les mouvements féministes ont été au cour des protestations des années 1970, et ils ont participé de la remobilisation perceptible, en France, à partir du milieu des années 1990. Il existe en outre, en France comme ailleurs, de nombreux mouvements féminins (qui ne s'affichent pas nécessairement comme « féministes»), pour certains constitués au début du siècle dernier, autour de causes civiques, confessionnelles, professionnelles ou encore d'enjeux locaux. Sans doute, de chaque côté de l'Atlantique, les centres d'intérêt des sociologues des mouvements sociaux reflètent-ils en partie les catégories nationales de l'entendement politique: en France, la catégorie des femmes, de même que le discours féministe et plus généralement ce que les Américains appellent la politique de l'identité (identity politics) (mobilisations de minorités fondées sur le genre, la sexualité ou la « race »/ethnicité), ont une bien plus faible légitimité politique qu'aux États-Unis. Dans le contexte français, le quasi-monopole des enjeux de classe et de redistribution matérielle dans la définition des mobilisations contestataires « de gauche » a conduit à détourner le regard de ces mobilisations vues comme « identitaires» (ainsi que d'autres, comme les mobilisations homosexuelles et queer et les mobilisations menées au nom de l'identité ethno-raciale ${ }^{8}$ ). Par ailleurs, pour comprendre pourquoi le mouvement des femmes ne s'est pas imposé comme objet d'analyse légitime en France, il faut tenir compte du contexte de faible institutionnalisation des études féministes et sur le genre (qui s'opère plus difficilement et avec retard par rapport aux États-Unis), en particulier en science politique qui a abrité jusqu'à aujourd'hui la plupart des travaux sociologiques sur les mouvements sociaux: dans cette discipline, les recherches sur les rapports sociaux de sexe ont longtemps été plus rares et maintenues dans une plus grande marginalité qu'en sociologie ou en histoire par exemple.

C'est précisément à la faveur d'une nouvelle ère d'institutionnalisation des études sur le genre dans les années récentes que plusieurs

7/ Certains sociologues des mouvements sociaux en France ont abordé les mouvements des femmes de manière connexe (Spanou, 1991 ; Mathieu, 2001). Le militantisme féminin post-68 a par ailleurs fait l'objet d'une attention renouvelée ces dernières années (Achin, Naudier, 2010 ; Pagis, 2009).

8/ Les mouvements homosexuels en France ont surtout été étudiés par le biais des mobilisations contre le SIDA (Fillieule, 2001; Broqua, 2006). Si certaines mobilisations de migrants ont été étudiées dans une perspective de sociologie des mouvements sociaux (notamment Hmed, 2006), peu de croisements ont été opérés à ce jour entre ce domaine d'études et la sociologie des rapports sociaux de race et d'ethnicité, à l'exception de quelques travaux (Célestine, 2009 ; Laplanche-Servigne, 2011). 
thèses soutenues à partir de la moitié des années 2000 ont pris pour objet les mouvements des femmes et féministes, en sociologie et en science politique (Fayolle, 2005; Giraud, 2005 ; Cîrstocea, 2006 ; Jacquot, 2006; Bereni, 2007; Lépinard, 2007 ; Revillard, 2007; Stoffel, 2008; Marteu, 2009 ; Jacquemart, 2011 ; Rétif, 2011), ou encore en histoire (Della Sudda, 2007 ; Pavard, 2010) ${ }^{9}$. Le séminaire "Genre, féminismes et mobilisations collectives », dont est issu ce dossier, a été un des lieux de construction collective de ces approches articulant sociologie des mouvements sociaux, études des mouvements des femmes (à la croisée de la sociologie, de l'histoire et de la science politique) et études sur le genre. Ce dossier ${ }^{10}$ présente les travaux de certain-e-s de ses membres et quelques acquis de ce travail collectif.

L'article de Laure Bereni et Anne Revillard ouvre le dossier en proposant de tirer parti du potentiel «paradigmatique » de l'étude des mouvements des femmes et féministes pour la sociologie des mouvements sociaux. À partir d'une revue non-exhaustive de la littérature - essentiellement anglophone - sur cet objet, au croisement de plusieurs disciplines des sciences sociales (principalement la sociologie, l'histoire et la science politique), les auteures montrent que ce corpus de travaux ébranle trois frontières souvent excessivement rigidifiées par la sociologie des mobilisations collectives et du militantisme, aux États-Unis comme en France : entre privé et public, entre militantisme et non-militantisme, et entre mouvements et institutions.

Analysant la pratique des avortements par plusieurs organisations militant pour sa libéralisation dans les années 1970 (le Mouvement pour l'avortement libre et gratuit (MLAC), le Mouvement français pour le planning familial et Choisir), Bibia Pavard montre que les moyens de la lutte peuvent contribuer à «faire mouvement», c'està-dire à impulser et transformer des mobilisations. La « découverte " d'une nouvelle méthode pour pratiquer des avortements (la «méthode Karman », qui permet de réaliser des avortements sans dispositifs techniques lourds et sans anesthésie) a joué un rôle d'impulsion des mobilisations collectives autour de la cause de la

9/ Plusieurs thèses sont en cours sur le mouvement féministe français, notamment celles de Marion Charpenel sur les processus de construction d'une mémoire collective dans l'espace de la cause des femmes contemporain, et de Camille Masclet sur les conséquences biographique de l'engagement féministe dans les années 1970. Des thèses sont également en préparation sur les mouvements des femmes dans d'autres contextes nationaux, comme celles de Delphine Lacombe (sur le Nicaragua), de Sophie Grenade (sur la République Démocratique du Congo) ou de Marième N’Diaye (sur le Sénégal).

10/ Dossier dont le titre est un clin d'œil au travail magistral réalisé par Charles Tilly sur l'évolution du répertoire de l'action collective en France, La France conteste : de 1600 à nos jours (Tilly, 1986). 
libéralisation de l'avortement. Au-delà, l'irruption de cette nouvelle pratique protestataire a fonctionné, au moins pendant quelques années, comme le ciment de mobilisations disparates, contribuant à faire tenir ensemble des organisations politiques variées et des militant-e-s aux parcours politiques très divers (des médecins militants, qui contestent l'ordre médical, aux militantes du Mouvement de libération des femmes, qui luttent contre l'ordre patriarcal).

Également centré sur les mobilisations féministes des années 1970, et plus spécifiquement sur l'engagement des hommes dans ces mouvements, l'article d'Alban Jacquemart explore les conditions et les modalités de cet engagement largement «improbable ». Il montre à quel point le renouvellement des formes du militantisme féministe légitime, pour lequel la non-mixité devient une norme centrale, transforme les conditions auxquelles les hommes peuvent y participer. Dans cette configuration, le registre « humaniste ", selon lequel la lutte féministe est une composante d'une lutte politique plus large, et qui avait été le principal moteur de l'engagement des hommes jusque-là, cède progressivement la place à un registre «identitaire», par lequel des hommes s'engagent dans le féminisme pour contester et retravailler leur identité masculine assignée. À partir d'une exploration fine de trajectoires d'hommes engagés dans ces mouvements, il montre que ce renouvellement des formes légitimes du militantisme féministe a alors des effets non seulement sur le nombre d'hommes engagés mais également sur le profil social des militants.

L'article de Marion Charpenel prend pour objet les effets et réappropriations d'un «événement», l'assassinat de Sohane Benziane (brûlée vive à 17 ans par un jeune homme dans un local à poubelles à Vitry-Sur-Seine en 2002), dans l'espace de la cause des femmes en France. Elle montre que cet événement a d'abord rendu visibles des fractures au sein de cet espace, mettant en exergue une rupture générationnelle de plus en plus manifeste depuis la seconde moitié des années 1990. Dans un second temps, toutefois, cet évènement a été reconstruit par certaines militantes comme un catalyseur d'unité du mouvement féministe. Les opérations de cadrage à l'œuvre dans le travail de commémoration de l'événement ont joué un rôle clef dans ce processus. En particulier, les ruptures entre générations et catégories de femmes (femmes «blanches » des classes moyennes et supérieures versus jeunes femmes de classes populaires et descendantes de l'immigration postcoloniale) ont été provisoirement suspendues par la construction d'un rapport de sororité entre Simone de Beauvoir, figure tutélaire et largement consensuelle du féminisme 
de la seconde vague, et Sohane, désignée comme victime dramatique du sexisme dans un quartier populaire de la banlieue parisienne.

C'est la traduction originale d'extraits des deux premiers chapitres de Faithful and Fearless, ouvrage majeur de la sociologue américaine Mary Katzenstein, qui clôt le dossier. Dans ce livre, l'auteure prend pour objet les mobilisations féministes qui se sont déployées, aux États-Unis, au cour même de deux institutions particulièrement contraignantes, l'armée et l'Église catholique. Elle montre comment ces luttes, quoique contraintes dans leurs formes par les normes de fonctionnement de ces deux institutions, ont pu se déployer grâce à l'existence d'espaces protégés en leur sein, que Katzenstein qualifie d' « habitats institutionnels ». Cette analyse de la politique contestataire intra-institutionnelle la conduit, dans les extraits ici traduits, à proposer deux reformulations théoriques majeures pour la sociologie des mouvements sociaux. Il s'agit d'une part de substituer, à la dichotomie entre l'intérieur et l'extérieur des institutions, une analyse en termes de continuum prenant en considération les différentes facettes des liens de dépendance/allégeance (financière, organisationnelle, discursive) des militant-e-s à l'égard des institutions. D'autre part, Mary Katzenstein propose, à rebours des analyses assimilant institutionnalisation et déradicalisation, de désindexer la question du lieu de la contestation (en dedans ou en dehors des institutions) des questions ayant trait aux modes d'action et au contenu des revendications. Les contestations intra-institutionnelles peuvent ainsi être envisagées dans toute leur portée potentiellement subversive.

À partir d'une diversité de perspectives, ce dossier entend donc dépasser l'opposition entre approches françaises et américaines des mouvements sociaux, et mettre en lumière l'intérêt des mouvements des femmes comme terrain de constitution et d'épreuve empirique de nouveaux paradigmes théoriques pour l'étude des mobilisations collectives.

Laure Bereni

Centre Maurice Halbwachs, équipe PRO

(CNRS/EHESS/ENS)

laure.bereni@ens.fr

Anne Revillard

Centre de recherche sur l'action locale (CERAL)

Université Paris 13 - Villetaneuse

anne.revillard@gmail.com 


\section{RÉFÉRENCES BIBLIOGRAPHIQUES}

ACHIN C., NAUDIER D., 2010 Trajectoires de femmes « ordinaires » dans les années 1970. La fabrique de la puissance d'agir féministe, Sociologie, 1, p. 77-93.

AGRIKOLIANSKY E., FILLIEULE O., MAYER N., 2005 (dir.)

L'altermondialisme en France. La longue histoire d'une nouvelle cause, Paris : Flammarion.

AGRIKOLIANSKY E., FILLIEULE Penser les mouvements sociaux, Paris : La Découverte. O., SOMMIER I., 2010

AGRIKOLIANSKY E., SOMMIER I., Radiographie du mouvement altermondialiste, Paris : La 2005 (dir.) Dispute.

BANASZAK L.A., 2010

The Women's Movement Inside and Outside the State, Cambridge, New York : Cambridge University Press.

BANASZAK L.A., BECKWITH K., Women's Movements Facing the Reconfigured State, New RUCHT D., 2003 (dir.) York : Cambridge University Press.

BARD C., 1995

BECKWITH K., 2000

Les filles de Marianne. Histoire des féminismes 1914-1940, Paris : Fayard.

Beyond Compare? Women's Movements in Comparative Perspective, European Journal of Political Research, 37 , p. $431-468$

BERENI L., 2007

BROQUA C., 2006

De la cause à la loi. Les mobilisations pour la parité politique en France (1992-2000), Thèse de doctorat en science politique, université Paris 1, Panthéon-Sorbonne.

Agir pour ne pas mourir ! : Act Up, les homosexuels et le sida, Paris : Presses de Sciences po.

CELESTINE A., 2009

Mobilisations collectives et constructions identitaires. Le cas des Antillais en France et des Portoricains aux États-Unis, Thèse de doctorat en science politique, Institut d'études politiques de Paris.

CÎRSTOCEA I., 2006

Faire et vivre le postcommunisme. Les femmes roumaines face à la transition, Bruxelles : Éditions de l'Université de Bruxelles.

COMBES H., HMED C., MATHIEU L., SIMÉANT J., SOMMIER I., 2011

COSTAIN A. N., 1992

DELLA SUDDA M., 2007

Observer les mobilisations. Retour sur les ficelles du métier de sociologue des mouvements sociaux, Politix, 93, p. 7-27.

Inviting Women's Rebellion : a Political Process Interpretation of the Women's Movement, Baltimore : Johns Hopkins University Press.

Une activité politique féminine conservatrice avant le droit de suffrage en France et en Italie. Socio-histoire de la politisation des femmes catholiques au sein de la Ligue Patriotique des Françaises (1902-1933) et de l'Unione fra le donne cattoliche d'Italia (1909-1919), Thèse de doctorat en histoire, EHESS (Paris)/La Sapienza (Rome).

DUNEZAT X., 2004

Chômage et action collective. Luttes dans la lutte. Mouvements de chômeurs et chômeuses de 1997-1998 en Bretagne et rapports sociaux de sexe, Thèse de doctorat en sociologie, Université de Versailles-Saint-Quentin-en Yvelines.

FAYOLLE S., 2005

L'Union des femmes françaises : une organisation féminine de masse du parti communiste français, 1945-1965, Thèse de doctorat en science politique, université Paris 1 , Panthéon-Sorbonne. 
FERREE M., MUELLER C., 2004 Feminism and the Women's Movement : A Global Perspective, in : Snow D. A., Soule S. A., et Kriesi H. (dir.), The Blackwell Companion to Social Movements, Oxford : Blackwell, p. 576-607.

FILLIEULE O., 2001

Propositions pour une analyse processuelle de l'engagement individuel. Post scriptum, Revue française de science politique, 51 (1-2), p. 199-215.

FREEMAN J., 1973

The Origins of the Women's Liberation Movement, American Journal of Sociology, 78 (4), p. 792-811.

FREEMAN J., 1975

GARCIA GUADILLA N., 1981

The Politics of Women's Liberation, New York : Longman.

GIRAUD I., 2005

Libération des femmes. Le MLF, Paris : PUF.

Mouvements des femmes et changements des régimes genrés de représentation politique au Québec et en France 1965-2004, Thèse de doctorat en science politique, université de Montréal/université de Versailles-Saint-Quentin.

GUBIN E., et al., 2004 (dir.)

Le siècle des féminismes, Paris : Éditions de l'Atelier.

HMED C., 2006

JACQUEMART A., 2011

Loger les étrangers « isolés » en France. Socio-histoire d’une institution d'État : la Sonacotra (1956-2006), Thèse de doctorat en science politique, Université Paris 1, Panthéon-Sorbonne.

Les hommes dans les mouvements féministes français (1870-2010). Sociologie d'un engagement improbable, Thèse de doctorat en sociologie, EHESS, Paris.

JACQUOT S., 2006

L'action publique communautaire et ses instruments. La politique d'égalité entre les femmes et les hommes à l'épreuve du gender mainstreaming, Thèse de doctorat en science politique, Institut d'études politiques de Paris.

KATZENSTEIN M. F., 1998

Faithul and Fearless. Moving from Feminist Protest Inside the Church and the Military, Princeton : Princeton University Press.

KLEJMAN L., ROCHEFORT F., L L'Égalité en marche. Le féminisme sous la Troisième 1989 République, Paris : Presses de Science po.

LAPLANCHE SERVIGNE S., 2011 De l'expérience du racisme à sa politisation : mobilisations et représentation des «victimes » en France et en Allemagne aujourd'hui, Thèse de doctorat en science politique, Institut d'études politiques de Paris.

LÉPINARD É., 2007

L'égalité introuvable. La parité, les féministes et la République, Paris: Presses de Science po.

MARTEU E., 2009

Les associations de femmes arabes en Israël : actrices et enjeux de nouvelles formes de mobilisation palestinienne en Israël, Thèse de doctorat en science politique, Institut d'études politiques de Paris.

MATHIEU L., 2001

Mobilisations de prostituées, Paris : Belin.

MAURER S., 2001

Les chômeurs en action. Mobilisation collective et ressources compensatoires, Paris : L'Harmattan.

MCBRIDE D., MAZUR A., 2008

«Women's Movements, Feminism and Feminist Movements », in : G. Goertz et A. G. Mazur (dir.), Politics, Gender and Concepts, Cambridge : Cambridge University Press

MCADAM D., 1982

Political Process and the Development of Black Insurgency (1930-1970), Chicago : University of Chicago Press. 
MCADAM D., TARROW S. TILLY, C., 2001

MCCARTHY J., ZALD M., 1977

MOUCHARD D., 2005

MOUCHARD D., 2009

PAGIS J., 2009

PAVARD B., 2010

PECHU C., 2006

PICQ F., 1993

RETIF S., 2011

REVILLARD A., 2007

SAWICKI F., SIMÉANT J., 2009

SIMÉANT J., 1998

SPANOU C., 1991

STAGGENBORG S., 1998

STOFFEL S., 2008

TAYLOR V., 2005 [1989]

TAYLOR V., WHITTIER N., 1992 Collective identity in social movement communities :

TILLY C., 1978

TILLY C., 1986

TOURAINE A., 1982 (dir.) Lesbian feminist mobilization, in : Morris A. D. et Mueller C. M. (dir.), Frontiers in Social Movement Theory, New Haven : Connecticut, Yale University Press.

Dynamics of Contention, Cambridge : Cambridge University Press.

Resource Mobilization and Social Movements : A Partial Theory, American Journal of Sociology, 82 (6), p. 1212-1241.

Les altermondialismes (introduction), Critique internationale, 27, p. 129-130.

Être représenté : mobilisations d'exclus dans la France des années 1990, Paris : Economica.

Les Incidences biographiques du militantisme en Mai 68. Une enquête sur deux générations familiales: des "soixantehuitards » et leurs enfants scolarisés dans deux écoles expérimentales (Vitruve et Ange-Guépin), Thèse de doctorat en sociologie, EHESS, Paris.

Contraception et avortement dans la société française (1956-1979) : histoire d'un changement politique et culturel, Thèse de doctorat en histoire, Institut d'études politiques de Paris.

Droit au logement : genèse et sociologie d'une mobilisation, Paris : Dalloz/Nouvelle bibliothèque des thèses.

Libération des femmes : les années-mouvement, Paris : Seuil.

Genre et engagement associatif. Carrières et pratiques militantes dans six associations françaises et portugaises, Thèse de doctorat en science politique, Université de Rennes 1/Institut d'études politiques de Rennes.

La cause des femmes dans l'État: une comparaison FranceQuébec (1965-2007), Thèse de doctorat en sociologie, ENS Cachan.

Décloisonner la sociologie de l'engagement militant. Note critique sur quelques tendances récentes des travaux français, Sociologie du travail, 51 (1), p. 97-125.

La cause des sans-papiers, Paris : Presses de Sciences po.

Fonctionnaires et militants : étude des rapports entre l'administration et les nouveaux mouvements sociaux, Paris : L'Harmattan.

Social Movement Communities and Cycles of Protest : The Emergence and Maintenance of a Local Women's Movement, Social Problems, 45 (2), p. 180-204.

Représentation politique et institutionnalisation du féminisme. Le cas du Chili depuis la fin des années 1980. Thèse de doctorat en sciences politiques et sociales, Université libre de Bruxelles.

La continuité des mouvements sociaux. La mise en veille du mouvement des femmes, in : Fillieule O. (dir.), Le désengagement militant, Paris : Belin, p. 229-250.

From Mobilization to Revolution, New York : Random House.

La France conteste : de 1600 à nos jours, Paris : Fayard.

Mouvements sociaux d'aujourd'hui : acteurs et analystes, Paris : Éditions ouvrières. 